\title{
Stochastic differential equations and comparison of financial models with levy process using Markov chain Monte Carlo (MCMC) simulation
}

\author{
Kianoush Fathi Vajargah \\ Department of Statistics, Islamic Azad University, North branch, Tehran, Iran \\ E-mail: k_fathi@iau-tnb.ac.ir
}

Copyright () 2015 Kianoush Fathi Vajargah. This is an open access article distributed under the Creative Commons Attribution License, which permits unrestricted use, distribution, and reproduction in any medium, provided the original work is properly cited.

\begin{abstract}
An available method of modeling and predicting the economic time series is the use of stochastic differential equations, which are often determined as jump-diffusion stochastic differential equations in financial markets and underlier economic dynamics. Besides the diffusion term that is a geometric Brownian model with Wiener random process, these equations contain a jump term that follows Poisson process and depends on the type of market. This study presented two different models based on a certain class of jump-diffusion stochastic differential equations with random fluctuations: Black- Scholes model and Merton model (1976), including jump-diffusion (JD) model, which were compared, and their parameters and hidden variables were evaluated using Markov chain Monte Carlo (MCMC) method.
\end{abstract}

Keywords: Levy Process, Markov Chain Monte Carlo, Black-Scholes Model, Merton Model, Stochastic Differential Equations.

\section{Introduction}

One of the main discussions on trading option pricing theory in finance is the matter of determining or, more specifically, predicting the asset price at maturity date. Actually, if one decides to make an option contract of a deal (either as a seller or as a buyer of the option), he should estimate the asset price at maturity date and then decide to do so considering the economic factors, such as the risk rate and so forth. In this situation, price fluctuations in the capital market are of special importance. The models usually selected to study these fluctuations and do estimations and predictions contain stochastic processes and especially Levy process. A sample path of Levy process may include jumps in its own time period that are objective evidence of sudden changes or, in other words, a sudden rise or fall in asset price in the capital market. In this study, the researchers selected the two models based on Levy process, Black- Scholes model and Merton model, and compared differences between the two models according to the changes in the jump parameter of Merton model. Sample paths used in Black- Scholes model are jumpless and involve Brownian motion process (which is a special type of Levy process). However, other models, including Merton model, contain terms as jump generators that cause the result of Black- Scholes model to be different. In this study, the effect of such terms was examined in the difference of the final answer. The price at maturity date was estimated using Markov chain Monte Carlo (MCMC) method. The convergence rate of Monte Carlo in computation of integrals is $0\left(\mathrm{~N}^{-1 / 2}\right)$.

\section{Stochastic differential equations and Monte Carlo approximation}

In this simulation method, the expected values of $\mathrm{E}[\mathrm{g}(\mathrm{X}(\mathrm{T}))]$ for one answer, $\mathrm{X}$, are presented from a given stochastic differential equation with a given function $g$. Generally, the approximation error consists of two parts, random error and time-discretization error. The statistical error was estimated using the central limit theorem. The error estimation for 
time-discretization of Euler method directly with an additional remaining term that measures the $1 / 2$ order accuracy for strong approximation.

Consider the following stochastic differential equation:

$\mathrm{dX}(\mathrm{t})=\mathrm{a}(\mathrm{t}, \mathrm{X}(\mathrm{t}))+\mathrm{b}(\mathrm{t}, \mathrm{X}(\mathrm{t})) \mathrm{dW}(\mathrm{t})$

The value of $\mathrm{E}[\mathrm{g}(\mathrm{X}(\mathrm{T}))]$ can be calculated using Monte Carlo method for $\mathrm{t}_{0} \leq \mathrm{t} \leq \mathrm{T}$. Based on the MCMC method: $\mathrm{E}[\mathrm{g}(\mathrm{X}(\mathrm{T}))] \cong \sum_{\mathrm{j}=1}^{\mathrm{N}} \frac{\mathrm{g}\left(\overline{\mathrm{X}}\left(\mathrm{T} ; \omega_{\mathrm{j}}\right)\right)}{\mathrm{N}}$,

Where, $\bar{X}$ is an approximation of $\mathrm{X}$. based on Euler method, the error in Monte Carlo method is as follows: $[5,7]$

$\mathrm{E}[\mathrm{g}(\mathrm{X}(\mathrm{T}))]-\sum_{\mathrm{j}=1}^{\mathrm{N}} \frac{\mathrm{g}\left(\overline{\mathrm{X}}\left(\mathrm{T} ; \omega_{\mathrm{j}}\right)\right)}{\mathrm{N}}$,

$=E[g(X(T))-g(\bar{X}(T))]-\sum_{j=1}^{N} \frac{g\left(\bar{X}\left(T ; \omega_{j}\right)\right)-E[g(\bar{X}(T))]}{N}$

\subsection{Monte Carlo estimation and integration}

Assume the vector $\left(U_{1} \ldots \ldots U_{N}\right)$ and $U_{i} \sim u\left([0,1]^{d}\right)$ for $i=1 \ldots N$; the standard Monte Carlo estimation $I$ is defined as follows:

$\overline{\mathrm{I}}_{\mathrm{f}}=\overline{\mathrm{I}}_{\mathrm{N}}=\frac{1}{\mathrm{~N}} \sum_{\mathrm{i}=1}^{\mathrm{N}} \mathrm{f}\left(\mathrm{U}_{\mathrm{i}}\right)$

Regarding the law for large numbers:

$P\left(\lim _{\mathrm{N} \rightarrow \infty} \overline{\mathrm{I}}_{\mathrm{N}}=\mathrm{I}_{\mathrm{f}}\right)=1$

The Variance of $f(U)$ for the square integrable $f$ is as follows:

$\sigma_{\mathrm{f}}^{2}=\operatorname{var}[\mathrm{f}(\mathrm{U})]=\int_{[0,1]}\left(\mathrm{f}(\mathrm{x})-\mathrm{I}_{\mathrm{f}}\right)^{2} \mathrm{dx}$

Regarding the central limit theorem: [3]

$\overline{\mathrm{I}}_{\mathrm{N}}-\mathrm{I}_{\mathrm{f}} \rightarrow \mathrm{N}\left(0, \frac{\sigma_{\mathrm{f}}^{2}}{\mathrm{~N}}\right)$

\section{Quasi-random sequences}

The simplest example for the quasi-random sequences is the van der Corput sequence at the dimension $(\mathrm{d}=1)$. To produce this sequence, we write $\mathrm{n}$ in binary decimal. The nth point of $x_{n}$ is obtained reversing the digits of the decimal point in the opposite $\mathrm{n}$. Halton and Sobol sequences are other examples of the quasi-random sequences, whose algorithms are mentioned below.

\subsection{Halton sequence}

The Halton sequence is the most basic low discrepancy with multiple dimensions. This sequence is the expansion of van der Corput sequence in dimension d. The nth number of Halton sequence in one dimension for a prime base $p_{d}$ can be achieved through the following algorithm.

1) Write $\mathrm{n}$ as a number in base $p_{d}$ :

$n=\sum_{i=0}^{l} a_{i}(n) p_{d}^{i}=a_{0} p_{d}^{0}+a_{1} p_{d}^{1}+\ldots+a_{l} p_{d}^{l}$

2) Reverse the digits in decimal point. Write the number in base 10:

$\varphi_{p_{d}}(n)=\sum_{i=0}^{l} \frac{a_{i}(n)}{p^{i+1}}$

Generally, Halton sequence in dimension $\mathrm{d}$ is:

$x_{n}=\left(\phi_{2}(n), \phi_{3}(n), \ldots, \phi_{p_{d}}(n)\right)$

\subsection{Sobol sequences}

S-dimensional Sobol sequence for all dimensions uses the prime number 2. The first dimension of the Sobol' sequence is the van der Corput sequence in base 2, and the higher dimensions are variations of the sequence of the first dimension. To generate the $\mathrm{j}$-th component of Sobol sequence an initial polynomial of the degree $\mathrm{n}$ in $\mathrm{Z}$ is required.

$x^{n}+a_{1} x^{n-1}+a_{2} x^{n-2}+\mathrm{K}+a_{n-1} x+1$

Where the coefficients $a_{1}, \mathrm{~K}, a_{n-1}$ are all either 0 or 1 . The Sequence of positive integers is defined with with the following recursive equation: 
$m_{k}=2 a_{1} m_{k-1} \oplus 2^{2} a_{2} m_{k-2} \oplus \mathrm{K} \oplus 2^{n-1} a_{n-1} m_{k-n+1} \oplus 2^{s} m_{k-n} \oplus m_{k-n}$

Where $\oplus$ denotes a bit-by-bit addition. Initial values $\left\{m_{1}, m_{2}, \mathrm{~K}\right\}$ must be chosen so that each $m_{k}, 1<k<n$ is an odd number smaller than $2^{k}$. Positive numbers $\left\{v_{1}, v_{2}, \mathrm{~K}\right\}$ are defined as $v_{k}=\frac{m_{k}}{2^{k}}$. Then the $\mathrm{j}$-th components of the point $\mathrm{i}$ th or $x_{i j}$ in a Sobol sequence is:

$x_{i, j}=i_{1} v_{1, j} \oplus i_{2} v_{2, j} \oplus \mathrm{K}$

\section{Levy process}

Definition 4.1: The process $L=\{L(t): 0 \leq t<\infty\}$ compatible with the filter $\mathcal{F}_{t}$ and the condition $L(0)=0$ a.s. is called a Levy process when it contains independent stationary increments. In other words:

1) For each $0 \leq s<t<\infty, L(t)-L(s)$ is independent of $\mathcal{F}_{s}$.

2) For each $0 \leq s<t<\infty, L(t)-L(s)$ and $L(t-s)$ have the same distribution.

3) $L(t)$ is in continuous probability, that is:

$P\left\{\omega \in \Omega: \lim _{t \rightarrow s} L(t, \omega)=L(s, \omega)\right\}=1$

Theorem 4.1: (Levy decomposition theorem): If L is a Levi process; it can be decomposed as follows:

$L_{t}=B_{t}+\int_{\{|x|<1\}} x\left(N_{t}(., d x)-t v(d x)\right)$

$+t E\left[X_{1}-\int_{\{|x| \geq 1\}} x N_{1}(., d x)\right]+\int_{\{|x| \geq 1\}} x N_{t}(., d x)$

$=B_{t}+\int_{\{|x|<1\}} x\left(N_{t}(., d x)-t v(d x)\right)+\alpha t+\sum_{0<s \leq t} \Delta X(s) 1_{\{|\Delta X(s)| \geq 1\}}$

Where, B is a Brownian motion; for each set of $\Lambda$ in which $0 \notin \bar{\Lambda}, N_{t}^{\Lambda}=\int_{\Lambda} N_{t}(., d x)$ is a Poisson process independent of B; if $\Lambda$ and $\Gamma$ are separate from each other, $N_{t}^{\Lambda}$ and $N_{t}^{\Gamma}$ are independent, $N_{t}^{\Lambda}$ has the parameter $v(\Lambda)$, and $v(d x)$ is a measure on $\mathbb{R} \backslash\{0\}$, as $\int \min \left(1, x^{2}\right) v(d x)<\infty$.

Theorem 4.2: (Levy-Khintchine formula): Assume L is a Levy process with Levy measure of $v$, then: $E\left[e^{i u L_{t}}\right]=e^{-t \psi(u)}$

Where,

$\psi(u)=\frac{\sigma^{2}}{2} u^{2}-i \alpha u+\int_{\{|x| \geq 1\}}\left(1-e^{-i u x}\right) v(d x)+\int_{\{|x|<1\}}\left(1-e^{-i u x}+i u x\right) v(d x)$

Moreover, the unique distribution of Levy process is determined with the given $\mathrm{v}, \sigma^{2}$, and $\alpha$.

Simulation of Levy paths: Assume that $L=\left\{L_{t}: 0 \leq t \leq T\right\}$ is a jump-diffusion Levy process, that is, a combination of a Brownian motion and a compensated Poisson process, the sample paths of the process can be formulated as follows:

$L_{t}=b t+\sigma B_{t}+\left(\sum_{k=1}^{N_{t}} J_{k}-t \lambda k\right)$

Where, $b \in \mathbb{R}, \sigma \in \mathbb{R}^{+}$, and $B=\left\{B_{t}: 0 \leq t \leq T\right\}$ is a standard Brownian motion. Furthermore, $N=\left\{N_{t}: 0 \leq t \leq\right.$ $T\}$ is a Poisson process with the parameter $\lambda$, and thus, $E\left(N_{t}\right)=\lambda t$. Finally, $J=\left(J_{k}\right)_{k \geq 1}$ is a sequence of independent random variables with the same distribution as the probability function $\mathrm{F}$, as $E(J)=k<\infty$ and $\mathrm{F}$ describes the distribution of jumps according to the Poisson process of $N_{t}$. All the random components of the above structure are correspondingly independent. Each Levy process is determined with a unique triad (b, c, v) in which $b \in \mathbb{R}$ is the cumulative term, $c \in \mathbb{R}^{+}$is the diffusion coefficient, and $\mathrm{v}$ is the Levi measure.

\section{Financial models with levy process}

\subsection{The black Scholes model: it is the most famous model used for examining the changes in asset price through Levi process. This model assumes that $L_{1} \sim N\left(\mu, \sigma^{2}\right)$, that is:}

$f_{L_{1}}(x)=\frac{1}{\sqrt{2 \pi \sigma^{2}}} \exp \left\{-\frac{(x-\mu)^{2}}{2 \sigma^{2}}\right\}$

The characteristic function would be as follows:

$\varphi_{L_{1}}(u)=\exp \left\{i u \mu-\frac{\sigma^{2} u^{2}}{2}\right\}$

And also, $E\left(L_{1}\right)=\mu$ and $\operatorname{Var}\left(L_{1}\right)=\sigma^{2}$.

The canonical decomposition of Levy process in this study is as follows:

$L_{t}=\boldsymbol{\mu} \mathbf{t}+\sigma B_{t}$

It means that one should only perform the time-discretization on a given distance and simulate a Brownian path and add it to the factor $\mu t_{i}$ in order to simulate a Levy path as shown above. 
The triad of the Levi process characteristic is $\left(\mu, \sigma^{2}, 0\right) .[1,2]$

\section{Merton model}

the difference in the models only refers to the type of canonical decomposition of levy process. in Merton mode, the canonical decomposition is as follows

$L_{t}=\mu t+\sigma B_{t}+\left(\sum_{k=1}^{N_{t}} J_{k}\right)$

Where, $J_{k} \sim N\left(\mu_{J}, \sigma_{J}^{2}\right) \cdot k=1,2, \ldots$. Therefore, jump size distribution is with the density $f_{J}(x)=$ $\frac{1}{\sqrt{2 \pi \sigma_{J}^{2}}} \exp \left\{-\frac{\left(x-\mu_{J}\right)^{2}}{2 \sigma_{J}^{2}}\right\}$.

The triad of the Merton model with Levi process is $\left(\mu, \sigma^{2}, \lambda \times f_{J}\right)$.

As shown by the equations, the difference between Black-Scholes model and Merton model is the presence of an additional term $\left(\sum_{k=1}^{N_{t}} J_{k}\right)$ related to the jump in Levi process, in which $J_{k} \sim N\left(\mu_{J}, \sigma_{J}^{2}\right) ، k=1,2, \ldots$. To keep it simple, it is assumed that $J_{k} \sim N(0,0 / 01) ، k=1,2, \ldots$. It means that all $\mathrm{J}_{\mathrm{K}} \mathrm{S}$ are selected from the normal distribution with similar parameters. In this case, the jump size should be calculated at each discrete time. The size with a Poisson process and a random variable is proportional to the normal distribution. The Poisson process also is proportional to the length of discrete interval and a given Poisson rate. From the theoretical point of view, the smaller the Poisson process rate, the fewer the number of points would be in the sample paths where jumps occur. Moreover, if it is assumed that the Poisson process rate is fixed at a value, the jump size turning out of the normal distribution associates with certain mean and variance parameters. The numerical results have been provided for different values of the three parameters of Poisson process rate $\theta$, mean normal distribution $\mu$, and normal distribution variance $\sigma^{2}$. [10].

\section{Numerical examples and results}

\subsection{Black-Scholes model and Asian option pricing}

Consider a problem of Asian option pricing with an optional arithmetic mean, the final discounts for a European-Asian style will be:

Max (Save - K, 0)

Where $\mathrm{K}$ is the price when $\mathrm{T}$ is due and $S_{a v e}=\frac{1}{d} \sum_{j=1}^{d} S_{t_{j}}$ is the arithmetic average of the basic asset at intervals equal to $\Delta t, j=1 \ldots d$,

$\Delta \mathrm{t}=\mathrm{T} / \mathrm{d}$ and $\mathrm{t} 0=0$.

We assume that a Black-Scholes model for the basic assessment is as follows:

$d S_{t}=\mu S_{t} d_{t}+\sigma S_{t} d B_{t}$

Where $\mu$ is the basic average return and $\sigma$ is volatility and $B_{t}$ is the standard Brownian motion.

Based on the natural risk of pricing principle, the asset value at time zero is given as:

$C_{A}=E_{Q}\left[\mathrm{e}^{-\mathrm{rT}} \max \left(\mathrm{S}_{\text {ave }}-\mathrm{K}, 0\right)\right]$

Where EQ [.] is the hope of the normal risk $\mathrm{Q}$.

Let $\mu=r$ ( $r$ is the relative risk-benefit), so an analytical solution for formula (1) is as follows:

$\mathrm{S}_{\mathrm{t}}=\mathrm{S}_{0} \exp \left(\left(\mathrm{r}-\frac{1}{2} \sigma^{2}\right) \mathrm{t}+\sigma \mathrm{B}_{\mathrm{t}}\right)$

Simulating the Asian option pricing, we need to simulate Brownian motion.

We have shown that one standard method to generate Brownian motion with successive periods is as follows:

$B_{t_{j}}=B_{t_{j-1}}+\sqrt{\Delta t} Z_{j} j=1 \ldots d$

Where $\mathrm{Z} 1 \ldots \mathrm{Zd}$ are random variables independent on standard normal distribution. According to the above function, the Asian option pricing can be written as:

$\mathrm{C}_{\mathrm{A}}=\int_{\mathrm{C}^{\mathrm{d}}} \mathrm{e}^{-\mathrm{rt}} \max \left(0, \frac{1}{\mathrm{~d}} \sum_{\mathrm{j}=1}^{\mathrm{d}} \mathrm{S}_{0} \exp \left[\left(\mathrm{r}-\frac{\sigma^{2}}{2}\right) \mathrm{t}_{\mathrm{j}}+\sigma \sqrt{\Delta \mathrm{t}} \sum_{\mathrm{i}=1}^{\mathrm{j}} \phi^{-1}\left(\mathrm{x}_{\mathrm{i}}\right)\right]-\mathrm{K}\right) \mathrm{dx}$

Where $\phi($.$) is the standard normal distribution? The above integral is compared and simulated using the three MCMC$ sampling, Sobol and Halton sequences. 
Table 1: The Comparison of Three Methods for Asian Option Pricing Under the Sobol, Halton, MCMC Methods for: S0 $=100, \Sigma=0.3$, R $=0.05$, T $=1$, and $\mathrm{K}=70$.

\begin{tabular}{llll}
\hline Type of Simulation & Value of Option & Error & Std \\
\hline Sobol Sequence & 31.5581 & 0.1388 & 0.7082 \\
Halton Sequence & 31.6421 & 0.2887 & 1.4731 \\
MC MC & 28.5803 & 3.6231 & 1.8485 \\
\hline
\end{tabular}

In the above calculations, the simulation method under Sobol sequence is clearly more accurate than the Halton sequences and MCMC methods for determining the Asian option pricing. [4], [6].

\subsection{Levi process and MCMC simulation}

The asset price at maturity date should be estimated using the following equation:

$\mathrm{S}_{\mathrm{T}}=\mathrm{S}_{0} \mathrm{e}^{\mathrm{L}_{\mathrm{T}}}$

The Monte Carlo estimator is calculated using frequent simulations in a way that the sample paths of a Levi process, that is, $\mathrm{L}_{\mathrm{T}}$, is first simulated, then, different prices as estimates of the asset price at maturity date are obtained. The Monte Carlo estimator is obtained through repeating the above procedure for many times, 10000 times for example, and considering the mean value of the prices.

In [], the final answer of 38.9618 has been presented as the estimate of asset price at maturity date for the Equation 1-2 of Black-Scholes model with $\mu=1, \sigma=0.3$ and $\mathrm{S}_{0}=30$; and this answer is compared to the answer of Merton model for different parameters.

Table 2: The Table for Price Estimation of Merton Model For $\Theta=0.25$

\begin{tabular}{lllllll}
\multicolumn{8}{c}{ Table 2: The Table for Price Estimation of Merton Model For $\Theta=0.25$} \\
\hline \multirow{4}{*}{$\theta=0.25$} & $\sigma^{2}=1$ & $\sigma^{2}=0.1$ & $\sigma^{2}=0.01$ & $\sigma^{2}=0.001$ & $\sigma^{2}=0.0001$ \\
& $\mu=-1$ & 38.0282 & 37.4831 & 37.4076 & 37.4356 & 37.5352 \\
& $\mu=0$ & 40.1606 & 39.2335 & 38.8883 & 39.0133 & 38.9911 \\
& $\mu=1$ & 47.6568 & 43.5077 & 43.3318 & 43.0947 & 43.1306 \\
\hline
\end{tabular}

Table 3: The Table for Price Estimation of Merton Model For $\Theta=0.5$

\begin{tabular}{llllll}
\multicolumn{6}{c}{ Table 3: The Table for Price Estimation of Merton Model For $\Theta=0.5$} \\
\hline \multirow{4}{*}{$\theta=0.5$} & $\sigma^{2}=1$ & $\sigma^{2}=0.1$ & $\sigma^{2}=0.01$ & $\sigma^{2}=0.001$ & 36.1071 \\
& $\mu=-1$ & 36.8672 & 36.0540 & 36.0065 & 35.83053 \\
& $\mu=0$ & 40.589 & 39.2443 & 39.143 & 39.0371 \\
\hline
\end{tabular}

Table 4: The Table for Price Estimation of Merton Model For $\Theta=1$

\begin{tabular}{cllllll}
\hline & & $\sigma^{2}=1$ & $\sigma^{2}=0.1$ & $\sigma^{2}=0.01$ & $\sigma^{2}=0.001$ & $\sigma^{2}=0.0001$ \\
\hline \multirow{3}{*}{$\theta=1$} & $\mu=-1$ & 35.2784 & 33.4004 & 33.2568 & 33.0529 & 33.1667 \\
& $\mu=0$ & 39.544 & 39.3744 & 38.9357 & 38.9045 & 38.9970 \\
\hline
\end{tabular}

Table 5: The Table for Price Estimation of Merton Model For $\Theta=2$

\begin{tabular}{|c|c|c|c|c|c|c|}
\hline & & $\sigma^{2}=1$ & $\sigma^{2}=0.1$ & $\sigma^{2}=0.01$ & $\sigma^{2}=0.001$ & $\sigma^{2}=0.0001$ \\
\hline \multirow{3}{*}{$\theta=2$} & $\mu=-1$ & 31.9513 & 28.8133 & 28.1129 & 28.2560 & 28.3450 \\
\hline & $\mu=0$ & 42.1657 & 40.1572 & 39.0603 & 38.9937 & 39.0882 \\
\hline & $\mu=1$ & 205.3693 & 100.6305 & 90.4905 & 94.6427 & 90.9961 \\
\hline
\end{tabular}

The above tables present the answer of Merton model at 4 different levels of Poisson process rate $\theta, 3$ different levels of mean normal distribution $\mu$, and 5 different levels of normal distribution variance $\sigma^{2}$.

Firstly, the effect of different values of $\theta$ is examined. According to the figures on next pages, minimum differences at all 4 levels of $\theta$ are related to $\mu=0$ and the differences reduce with an increase in values of $\sigma^{2}$. However, as the values of $\theta$ get greater, the differences increase, especially at the level of $\mu=1$, and becomes stable at high values when the variance decreases. It means that as the Poisson process rate in the term relevant to the jump of Merton model increases, the answer differs with that of Black-Scholes model more than usual. If the jump size results from a normal distribution with zero mean and very small variance, it is justified in a different way. The Poisson process rate, that is, the time of jumps, is high, but no significant jump occurs in practice because the size of jumps in these times is very small. Otherwise, when the mean of jumps is not zero, or variance of jumps is not very small, the difference in answer is certainly significant.[8], [9]. 

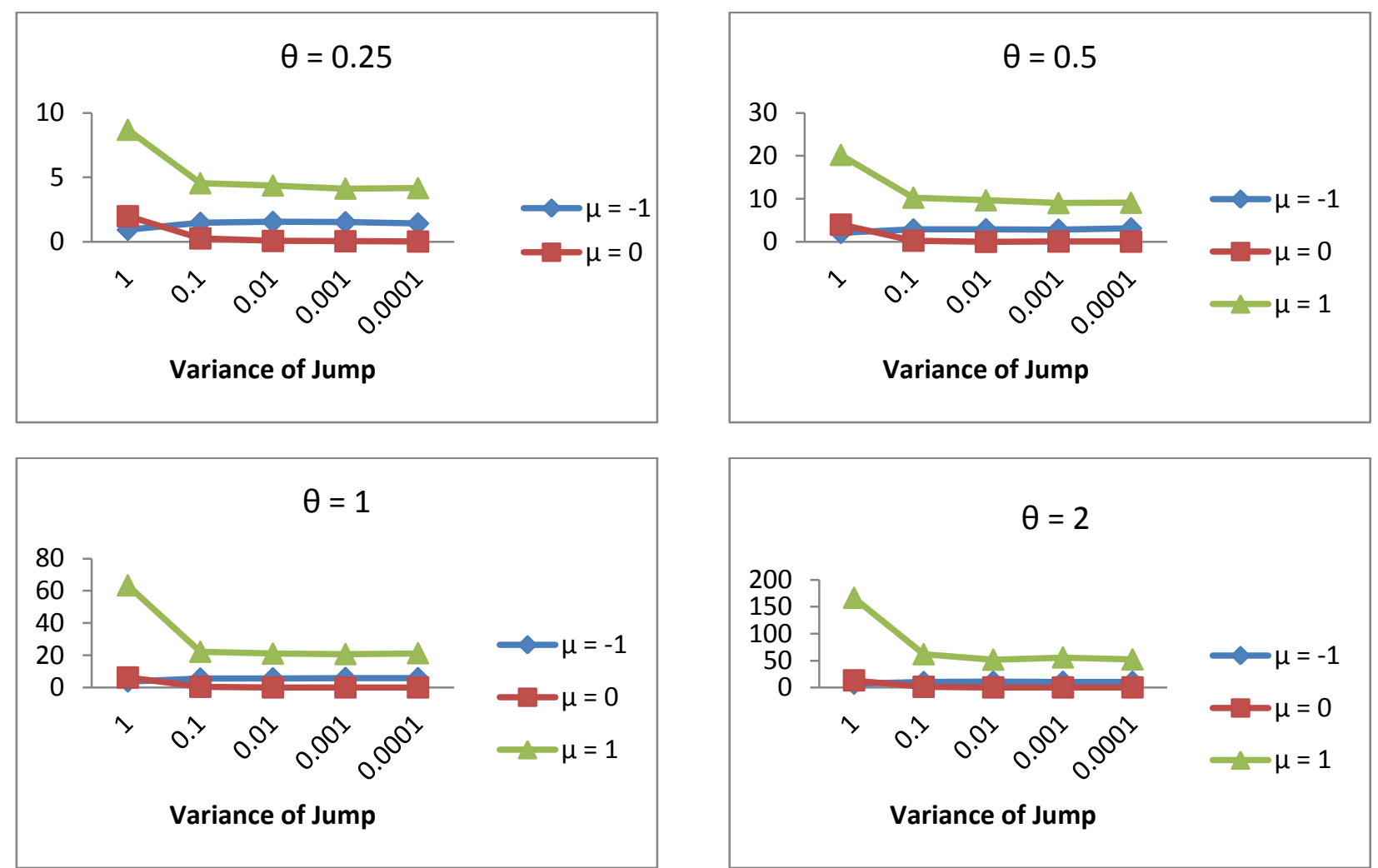

Fig. 1: The Effect of Different Values of $\mu$ And $\sigma^{2}$ at 4 Different Levels of $\Theta$.

The figures on next pages show the effect of different values of $\theta$ and $\sigma^{2}$ at 3 different levels of mean. Also in this case, minimum difference is obtained for $\mu=0$; the optimum state at this level occurs in lower values of $\theta$; and the differences reduce with a decrease in variance.
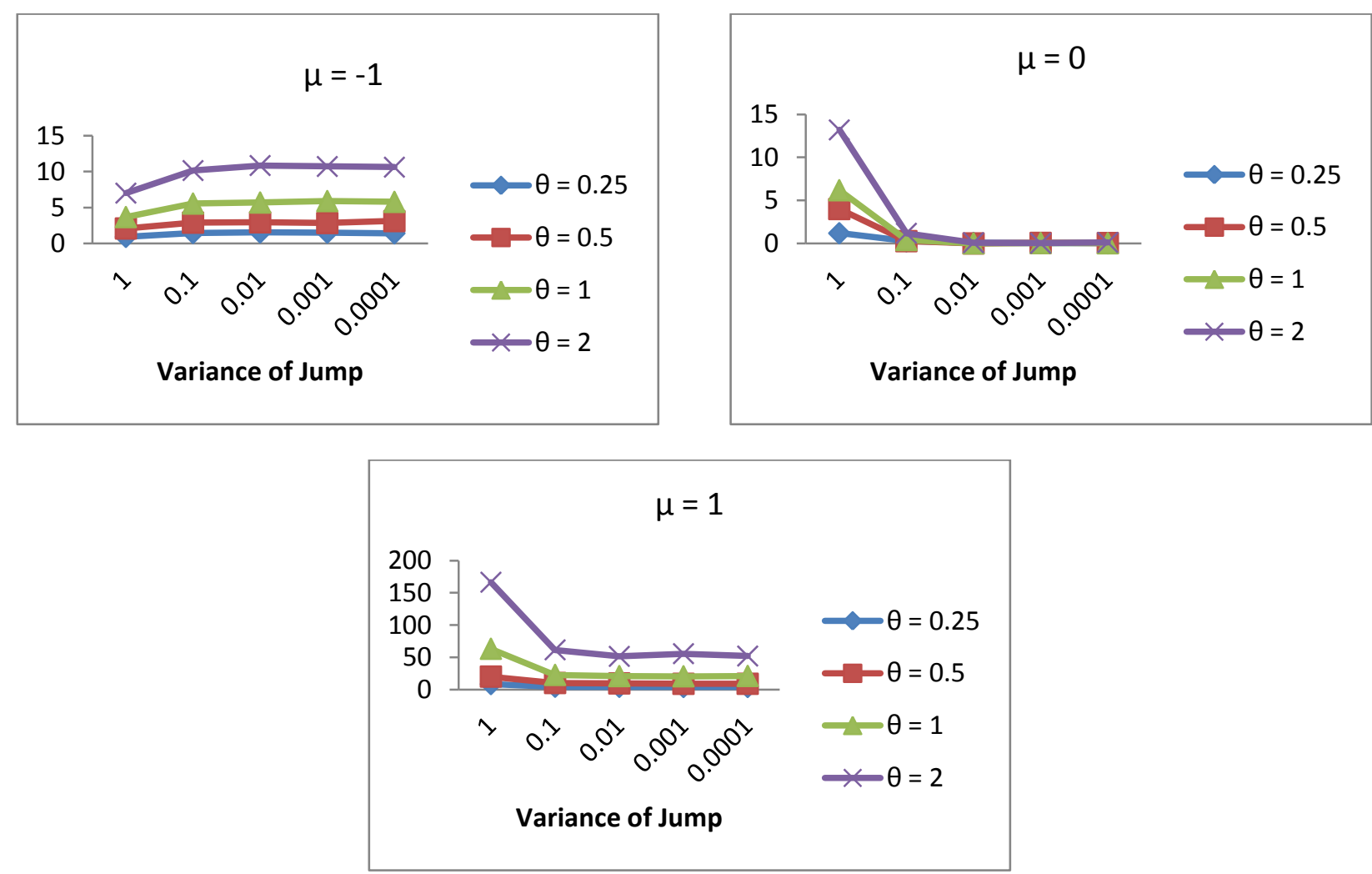

Fig. 2: The Effect of Different Values of $\Theta a n d ~ \sigma^{2}$ at 3 Different Levels of Mean. 
The figures on next pages examines the effect of different values of $\theta$ and $\mu$ at different levels of $\sigma^{2}$; as shown by the figures, minimum difference is obtained for the smallest value of $\theta$ and $\mu=0$ and at lowest level of variance, that is, $\sigma^{2}=0.0001$
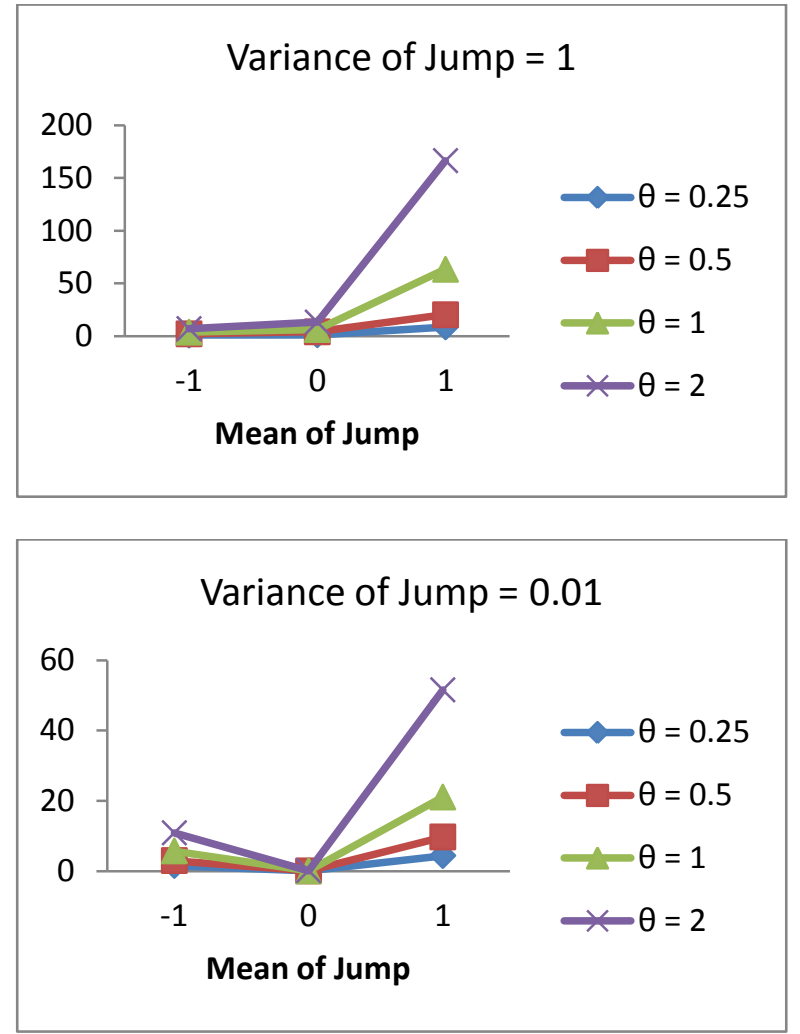
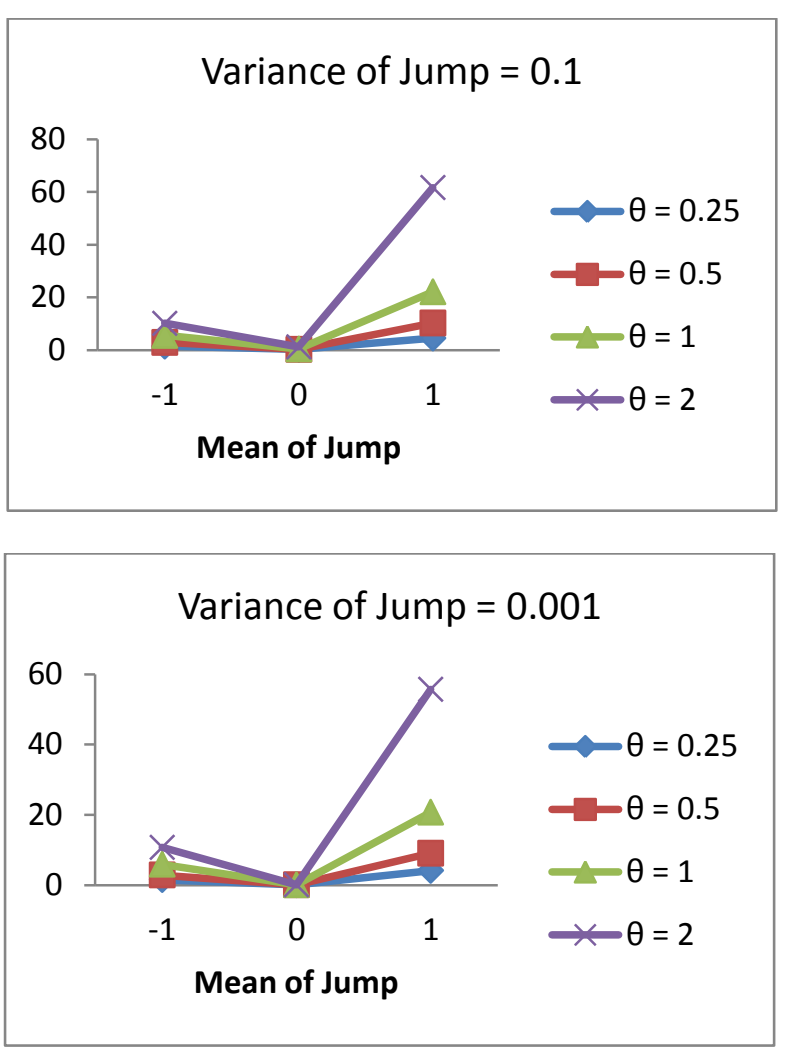

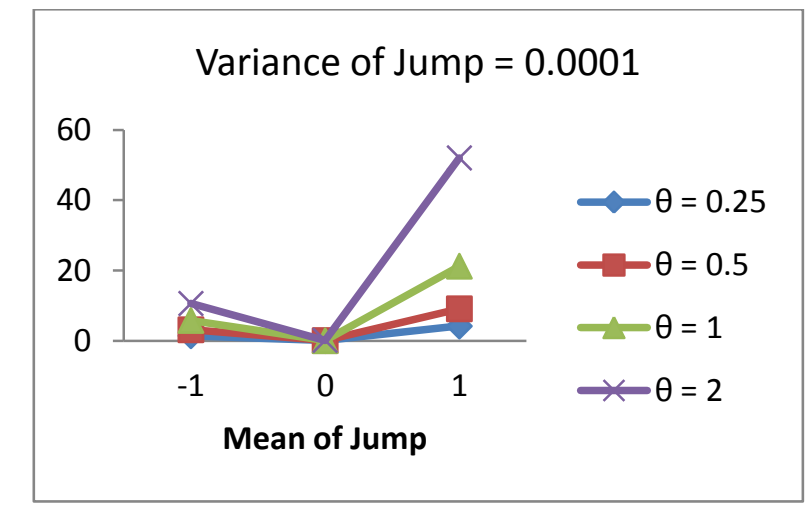

Fig. 3: The Effect of Different Values of $\Theta$ and $\mu$ at 5 Different Levels of $\sigma^{2}$

\section{Conclusion}

When the Poisson rate in Merton model is small, or the size of jumps results from a normal distribution with zero mean and very small variance, the jumps can be totally discarded, and the difference between Black-Scholes model and Merton model is ignorable. In this case, any difference associates with the stochastic nature of the system and simulation of sample paths. Moreover, when the Poisson process rate is very small, that is, most of the simulated values of jump times are 0 , or in other words, the probability for occurrence of jumps in the sample path is almost zero, obviously jumps cannot occur even if the size of jumps is not zero. Therefore, there is no difference between the two models in this case as well. A significant difference between the two models appears only when the Poisson process rate is large to the extent that jumps occur in the sample path at certain times, and the jump size at these times is resulted from a normal distribution with a non-zero mean or a large variance. 


\section{References}

[1] D.Applebaum, 2004, Levy Process and Stochastic Calculus, Cambridge University Press. http://dx.doi.org/10.1017/CBO9780511755323.

[2] J.Bertion, 1998, Levy Process, Cambridge University Press.

[3] P.Billingsley, 1995, Probability and Measures, 3rd ed, Wiley\&Sons, New York.

[4] R.Cont, P.Tankov, 2004, Fainancial Modeling With Jump Processes, Chapman\&Hall/CRC Press.

[5] J.S.Dagpunar, 2007, Simulation and Monte Carlo With Application in Finance and MCMC,Wiley\&Sons. http://dx.doi.org/10.1002/9780470061336.

[6] S.Dereich, F, Heidenreich, 2011, a Multilevel Monte Carlo Algorithm for Levy-Driven Stochastic Differential Equqtial Equations, Stochastics Processes and Their Applications, 121:1565-1587. http://dx.doi.org/10.1016/j.spa.2011.03.015.

[7] M.B.Giles, Multi-level Monte Carlo Path Simulation, 2008, Operations Reserch, 56 (3):607-617. http://dx.doi.org/10.1287/opre.1070.0496.

[8] M.B.Giles, B.J.Waterhouse, 2009, Multilevel Quasi-Monte Carlo Path Simulation,Radon Series Comp.Appl.Math8,1-18.

[9] D.Henderson, P.Plaschko, 2006, Stochastic Differential Equations In Scientific Publishing,Singapore.

[10] S.Heston, 1993, A Closed-fom Solutions Options with Stochastic Volatility with Applications to Bond and Currency Option, The Review of Finance Studies, 6(2):327-343. http://dx.doi.org/10.1093/rfs/6.2.327. 